\title{
Energy Harvesting Methods in Wireless Sensor Network: A Review
}

\author{
Samriti Thakur \\ M.Tech (Scholar) \\ CGC, Landran
}

\author{
Devendra Prasad, PhD \\ Professor \\ CGC, Landran
}

\author{
Amit Verma, PhD \\ Professor \\ CGC, Landran
}

\begin{abstract}
Wireless Sensor Networks (WSNs) are composed of small sensor nodes, (SNs) capable of gathering, communicating and data-processing. The energy consumption defines a challenge in several years. Sensor nodes, as components of wireless sensor networks, are battery ambitious devices and operate on an extremely frugal energy reasonable. In this paper surveys several methods used in today's wireless sensor networks with order to exceed the issue of energy consumption, power management and harvesting. WSNs are highly prone to energy deficiency issues. Since the star of the wireless technology the need of a set-up which is very efficient in terms of power was required. Because of wireless performance of wireless sensors network it is very necessary that we make out routing methods and protocols very efficient. Major scope of work is very important aspects of the energy harvesting thus also become very significant.
\end{abstract}

\section{Keywords}

Wireless Sensor Network, Energy harvesting, Power management and techniques of harvesting.

\section{INTRODUCTION}

The collection of insignificant, in-expensive wireless sensor network nodes, organized in networks groups and networks organized over a terrestrial area, proficient to integrate incessant and un-obtrusive quantity, computing and wireless communication, have concerned much consideration through the last period in forming the perception of smart spaces[1].One of the many experiments related with sensing numerous parameters from the situation, by using wireless sensor networks, is to how to communicate data and power the sensors. Batteries deliver the power source of sensor nodes [2]. to replacement or recharging. For disposition with hundreds of sensors, this means that a battery will need a standby every few days, what represents an inappropriate rate for many applications. Numerous solutions to the power problematic exist, such as reducing power consumption to the point where batteries can extend the sensor module's lifetime.

The rapid growth in difficulties for figuring everywhere has made processor a pivotal component of human mankind daily lives. Whether we use the processors to gather info from the Web, to apply them for entertaining purposes or to use them for running businesses, computers are noticeably attractive more extensive, mobile and smaller in size. What we often supervise and did not notice is the attendance of those billions of small inescapable computing devices around us which deliver the intelligence being combined into the real world [3].These prevalent computing devices can help to solve some critical difficulties in the events of our daily lives. When several wireless sensor nodes are interconnected together, they form a network called the Wireless Sensor Network. Sensor nodes agreed in network form will absolutely exhibition more and better characteristics than individual sensor nodes.

Rest of the paper is organized as follows, section 2. Describe the related work about the smart environment with pervasive computing, section 3. Describe the wireless sensor network and architecture, section 4. Describe about the energy harvesting, section 5. Describe about the benefits of energy harvesting, section 6. Describe about techniques of energy harvesting, and at last conclusion is made in section 7.

\section{SMART ENVIRONMENT WITH PERVASIVE COMPUTING}

Smart environment is among the emerging technologies expected to succeed in the common computing situation of the future [4].The concept of smart environment is becoming a reality with inescapable computing as well as progressions of various connected technologies such as wireless networking, micro-fabrication and addition using micro-electromechanical system technology and entrenched intelligent with microprocessors. Smart environments characterize the next evolutionary expansion step in various application areas such as construction, utilities, industrial, home, marine, animal habitat, traffic, etc. Like any sensitive organism, the smart situation relies first and foremost on corporeal data from the real world. Sensory data comes from multiple sensors of different modalities in dispersed locations. Similarly for the smart situation, information about its surroundings is also desirable just like what is arrested by the receptors in the organic systems. The information needed by the smart situations is provided by the dispersed WSN which has its pervasive sensor nodes for sensing, dispensation and communicating the material to the base station [5].To facilitate smart situations in various application areas, a general architecture of the data acquisition and distribution network is providing in Figure.2.

The data acquisition network is calculated to gather real-world information as well as to monitor the disorder of the targeted application. Data are collected at the base station in a wireless method, pre-processed and then dispersed to the end users via different interactive devices [6]. 


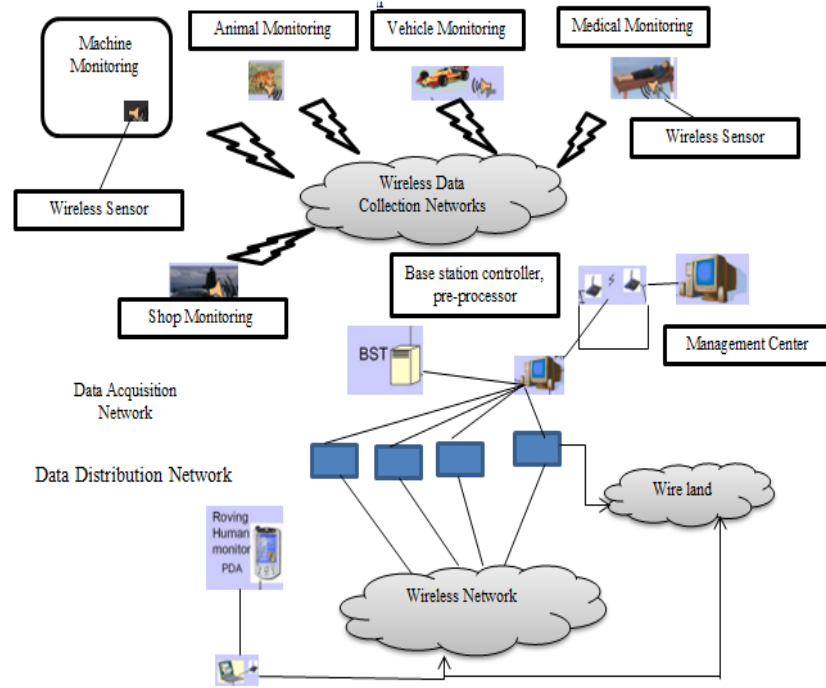

Figure 2: A general architecture of the data Acquisition and Distribution Network [6].

\section{WIRELESS SENSOR NETWORK AND ARCHITECTURE}

WSN can be designed of military applications such as battlefield surveillance and intrusion detection. The previous endeavors to build efficient military sensor networks as well as the fast developments in microelectronic design and wireless communication, WSN are gradually introduced to many civil application areas. With the incessant dedications of academic researchers and industrial researchers, people are getting closer and closer to the essential points to understand WSN technology.

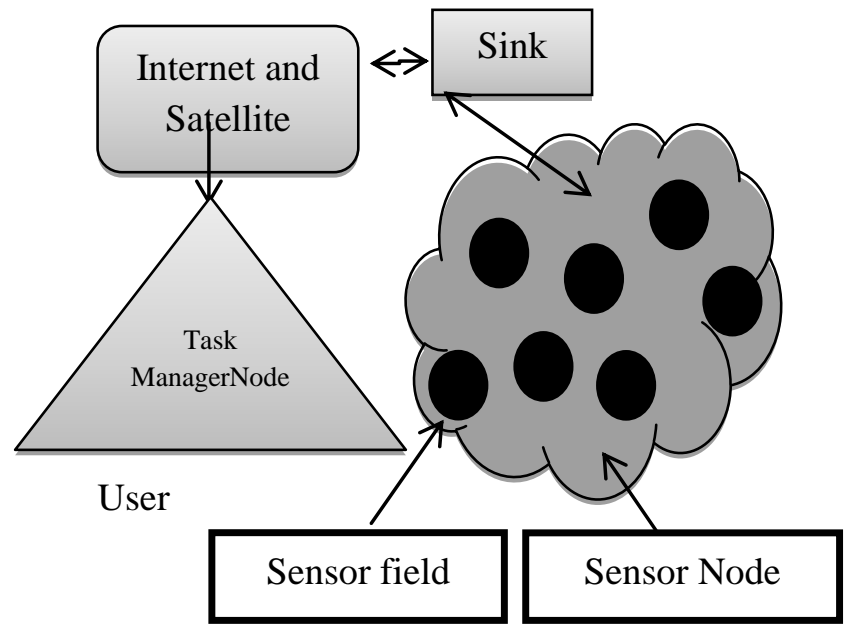

Figure 3: Architecture of Wireless Sensor Network.

The unique characteristics of WSN make it advantageous over the former networks on one hand, but on the other hand, many challenges are inevitable. Hence further research and thorough reflections on WSN are greatly needed [7].The architecture of a WSN typically consists of multiple pervasive sensor nodes, sink, public networks, manager nodes and end user. Many small, smart and in-expensive sensor nodes are scattered in the target sensor field to collect data and route the useful information back to the end user. These sensor nodes cooperate with each other via wireless connection to form a network and collect, disseminate and analyze data coming from the environment.
As illustrated in Figure.3, the data collected by node A is routed within the sensor field by other nodes. The data will reach the boundary node $\mathrm{E}$ and then be transferred to the sink. The sink serves as a gateway with higher processing capacity to communicate with the task executive node. The assembly between sink and task manager node is the public networks in the form of Internet or satellite. The end user will receive the data from the task manager node and perform some processing on these received data [8].

The WSN protocol stack shown in Figure. 4 consists of five network layers namely physical, data link, network, transport and application layers and three new elements: power management plane, mobility management plane and task management plane.

Starting from the lowest level, the physical layer is to meet the needs of receiving and transferring data collected from the hardware. It is well known that long distance wireless communication can be expensive, in terms of both energy and implementation complexity. The physical layer for WSNs,[9] energy minimization is considered significantly more important over and above the other factors like propagation and fading effects. Energy-efficient corporeal layer solutions are currently being followed by researchers to design for tiny, low-power, low-cost transceiver, sensing and processing units. The next higher layer is the data link layer which ensures reliable point-to-point and point-to-multipoint connections for the multiplexing of data torrents, data frame detection, medium access and error controller in the WSN. The data link layer should be power-aware and at the same time to minimize the collisions between neighbors' signals because the environment is noisy and sensor nodes themselves are highly mobile.

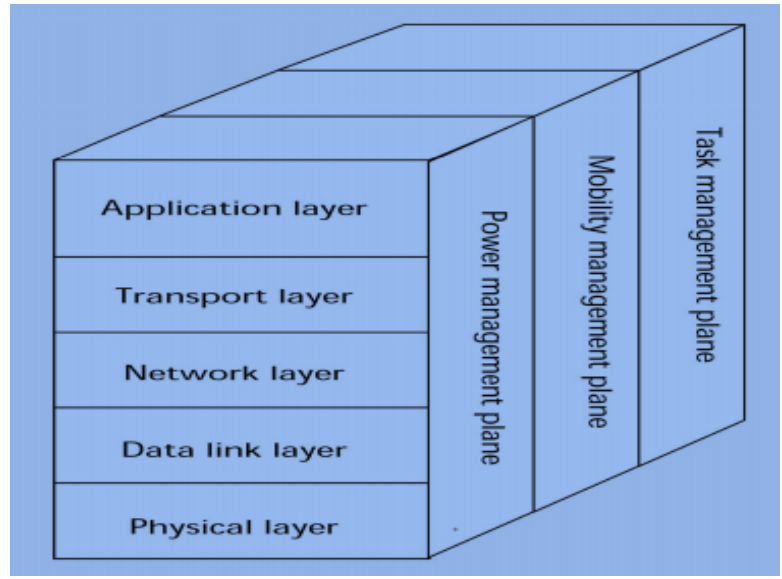

Figure 4: WSN Protocol Stack [9].

\section{ENERGY HARVESTING}

Energy harvesting is a method that captures, harvest or search unused ambient energy (such as vibrational, thermal, wind, solar, etc.) and converts the harvested energy into usable electrical energy which is deposited and used for performing sensing or actuation. The gathered energy is generally very small as compared to that large-scale energy harvesting using renewable energy sources such as solar farms and wind farms. Unlike the large-scale power stations which are fixed at a given location, the small-scale energy sources are portable and readily available for usage. Energy harvested from the ambient are used to power small autonomous sensors that are deployed in remote locations for sensing or even to endure long-term experience to hostile environments. The operations of these small independent sensors are often restricted by the 
reliance on battery energy. Hence the driving force behind the search for energy harvesting practice is the desire to power wireless sensor networks and moveable devices for extended operation with the supplement of the energy storage elements if not completely eliminating the storage elements such as batteries[10].

The impression of energy harvesting in comparative to wireless sensor network (WSN) entails the idea of scavenging energy from mechanical, vibrational, rotational, solar or thermal means rather than relying on mains power or alkaline/rechargeable batteries to power the sensor nodes in the WSN. For instance, power can be harvested from the mechanical force of a conventional mechanical ON and OFF switch being turned on or off. Alternately, power can be resultant from the alteration in temperature between the human body and the surrounding ambient environment. Energy harvesting is increasingly gaining notice in the WSN research as well as industry market because it is a very potential solution to extend the lifetime of the sensor node's operation [11].

\section{ADVANTAGES OF ENERGY HARVESTING}

Energy harvesting provides numerous benefits to the end user and some of the major benefits about EH suitable for WSN are stated and elaborated in the following list. Energy harvesting solutions can:

(1) Reduce the dependency on battery power. With the advancement of microelectronics technology, the power consumption of the sensor nodes are receiving lesser and lesser, hence harvested ambient/conservational energy may be sufficient to eliminate battery completely [12]

(2) Reduce installation cost. Self-powered wireless sensor nodes do not require power cabling and channels, hence they are very easy to install and they also reduce the heavy installation cost.

(3) Reduce maintenance cost. Energy gathering allows for the sensor nodes to function unattended once organized and eliminates service visits to replace batteries.

(4) Provide identifying and actuation capabilities in hard-to-access hazardous surroundings on a continuous basis.

(5) Provide long-term solutions. A reliable selfpowered sensor node will remain functional virtually as long as the ambient energy is available. Self-powered sensor nodes are seamlessly suited for long-term applications watching at decades of monitoring.

\section{METHODS OF ENERGY HARVESTING}

In both academic research works and industry applications, there are many research and development works being carried out on harnessing large-scale energy from various renewable energy sources such as solar, wind and water/hydro NREL (2010)[15]. Little attention has been paid to small-scale energy gathering methods and strategies in the past as there are hardly any need. Having said that, it does not mean that there is no research activity being conducted on small-scale energy harvesting. In fact, there are quite a significant amount of research works recorded in the literature that discuss about scavenging or harvesting small-scale environmental energy for low powered mobile electronic devices especially wireless sensor nodes. Energy harvesting research prototypes for harvesting various energy sources have been discussed. It describes the extraction of energy from kinetic motion. Roundy gave a comprehensive examination on vibration energy scavenging for wireless sensor network.

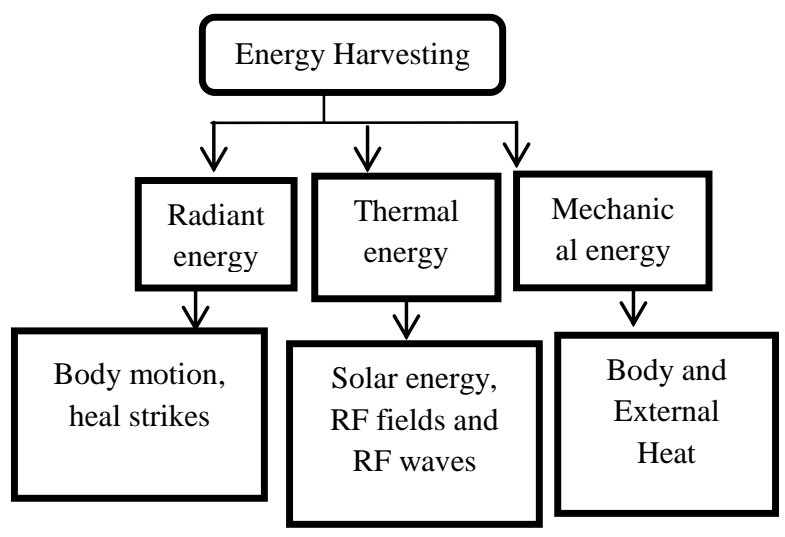

Figure 6: Methods of Energy Harvesting.

Figure.6 shows numerous types of ambient energy forms appropriate for energy harvesting along with examples of the energy sources. The energy types are thermal energy, radiant energy and mechanical energy [13].

Some energy harvesting research prototypes for gathering various liveliness sources have been discussed. A substantial piece of the research work describes the extraction of energy from kinetic motion. Roundy gave a comprehensive examination on vibration energy scavenging for wireless sensor network. There are other vibration based energy harvesting research works being reported for instances piezoelectric generators, wearable electronic textiles and electromagnetic vibration-based micro generator devices for intelligent sensor systems. In the research area of thermal energy harvesting, consider the system design aspects for thermal energy scavenging via thermoelectric conversion that exploits the natural temperature difference between the ground and air. Similarly, it has considered thermal energy harvesting through thermoelectric power generation from body heat to power wireless sensor nodes. Research on smallscale wind energy harvesting has also been performed by several groups of researchers [14].

\section{CONCLUSION}

The major hindrances of the "deploy and forget" nature of the wireless sensor networks (WSNs) are the imperfect energy capacity and unpredictable lifetime presentation of the battery. In order to overcome these problems, energy harvesting, this harvests energy from a variety of ambient energy foundations and converts into electrical energy to refresh the batteries. And also emerged a promising technology. In this paper, we reviewed two of the most used techniques in Energy harvesting in Wireless Sensor Networks. The shaking energy harvesting method is very efficient system and utilizes ambient vibrations to harvest energy. The thermal energy harvesting technique is also very powerful technique in energy harvesting. and utilizes See beck effect using thermocouples to generate energy. Both techniques have their qualities and disadvantages based on efficiency, cost, robustness etc. Thermal based system is highly robust has it has no mechanical part. Vibration based system has developed competence than thermal based system since Carnot efficiency has an upper limit on heat energy that can be recovered. 


\section{REFERENCES}

[1] Tan, Yen Kheng, and Sanjib Kumar Panda. "Review of energy harvesting technologies for sustainable wireless sensor network." Sustainable wireless sensor networks (2010): 15-43

[2] Habibu, Hussaini, Adamu Murtala Zungeru, Ajagun Abimbola Susan, and Ijemaru Gerald. "Energy harvesting wireless sensor networks: design and modeling." International Journal of Wireless \& Mobile Networks 6, no. 5 (2014): 17

[3] Akbari, Saba. "Energy harvesting for wireless sensor networks review." In Computer Science and Information Systems (FedCSIS), 2014 Federated Conference on, pp. 987-992. IEEE, 2014.

[4] Basagni, Stefano, M. Yousof Naderi, Chiara Petrioli, and Dora Spenza. "Wireless sensor networks with energy harvesting." Mobile Ad Hoc Networking: The Cutting Edge Directions (2013): 701-736.

[5] Niyato, Dusit, Ekram Hossain, Mohammad M. Rashid, and Vijay K. Bhargava. "Wireless sensor networks with energy harvesting technologies: A game-theoretic approach to optimal energy management." IEEE Wireless Communications 14, no. 4 (2007): 90-96.

[6] Lee, Vincent Chunwan. "Energy harvesting for wireless sensor networks." PhD diss., University of California, Berkeley, 2012.

[7] Li, Yin, and Ronghua Shi. "An intelligent solar energyharvesting system for wireless sensor networks." EURASIP Journal on Wireless Communications and Networking 2015, no. 1 (2015): 1.

[8] Gilbert, James M., and Farooq Balouchi. "Comparison of energy harvesting systems for wireless sensor networks." international journal of automation and computing 5, no. 4 (2008): 334-347
[9] Jindal, Ishu, and Er Divya Bharti. "A Review on Energy Harvesting Techniques in Wireless Sensor Networks." "International Journal for Science and Emerging Technologies with Latest Trends" 22(1): 11-18(2015) ISSN No. (Print): 2277-8136.

[10] R. C. Shah and J. M. Rabaey, "Energy aware routing for low energy ad hoc sensor networks," in Proc. IEEE Wireless Communications and Networking Conference (WCNC), Orlando, FL, 2002, pp. 350-355.

[11] M. Younis, M. Youssef, and K. Arisha, "Energy-aware routing in cluster-based sensor networks," in Proc. 10th IEEE/ACM International Symposium on Modeling, Analysis and Simulation of Computer and Telecommunication Systems, 2002.

[12] J. A. Paradiso and T. Starner, "Energy scavenging for mobile and wireless electronics," Pervasive Computing, IEEE, vol. 4, pp. 18-27, 2005.

[13] K. Vijayaraghavan and R. Rajamani, "Active Control based Energy Harvesting for Battery-less Wireless Traffic Sensors: Theory and Experiments," in Proc. of American Control Conference, NY, USA, July 11-13 2007, pp. 4579-4584

[14] J. Paulo and P.D. Gaspar "Review and Future Trend of Energy Harvesting Methods for Portable Medical Devices" Proceedings of the World Congress on Engineering, pp. 909-914, 2010.

[15] Stoj, Mile K., and Mirko R. Kosanovi. "Power management and energy harvesting techniques for wireless sensor nodes." In Telecommunication in Modern Satellite, Cable, and Broadcasting Services, 2009. TELSIKS'09. 9th International Conference on, pp. 65-72. IEEE, 2009. 